\title{
Micafungin: an evidence-based review of its place in therapy
}

This article was published in the following Dove Press journal:

Core Evidence

25 February 2014

Number of times this article has been viewed

\author{
Pola de la Torre \\ Annette C Reboli' \\ 'Cooper Medical School of Rowan \\ University, Camden, NJ, USA
}

\begin{abstract}
Invasive fungal infections have increased throughout the world. Many of these infections occur in patients with multiple comorbidities who are receiving medications with the potential for interactions with antifungal therapy that could lead to renal and hepatic dysfunction. The second marketed echinocandin, micafungin, was approved in 2005 for the treatment of esophageal candidiasis and prophylaxis of invasive Candida infections in patients undergoing hematopoietic stem cell transplantation. The indication for use was later expanded to include candidemia, acute disseminated candidiasis, Candida abscesses, and peritonitis. Like other echinocandins it is fungicidal against Candida species, including those that are polyene- and azole-resistant and fungistatic against Aspergillus species. Its formulation is by the intravenous route only and it is dosed once daily without a loading dose as $85 \%$ of the steady state concentration is achieved after three daily doses. It has a favorable tolerability profile with no significant drug interactions and does not need adjustment for renal or hepatic insufficiency.
\end{abstract}

Keywords: echinocandin, micafungin, Candida, Aspergillus, prophylaxis

\section{Core evidence clinical impact summary for micafungin}

\begin{tabular}{|c|c|c|}
\hline $\begin{array}{l}\text { Outcome } \\
\text { measure }\end{array}$ & Evidence & Implications \\
\hline \multirow[t]{4}{*}{$\begin{array}{l}\text { Disease-oriented } \\
\text { evidence }\end{array}$} & $\begin{array}{l}\text { I. Demonstrates excellent } \\
\text { efficacy for the treatment of } \\
\text { candidemia/invasive candidiasis. }\end{array}$ & $\begin{array}{l}\text { I. May be used as front-line } \\
\text { therapy in treating candidemia/ } \\
\text { invasive candidiasis, except for } \\
\text { meningitis. }\end{array}$ \\
\hline & $\begin{array}{l}\text { 2. Demonstrates good efficacy } \\
\text { as primary or salvage therapy } \\
\text { in high-risk patients with } \\
\text { invasive aspergillosis. }\end{array}$ & $\begin{array}{l}\text { 2. Numbers are small in those } \\
\text { treated with micafungin } \\
\text { monotherapy. The major role } \\
\text { remains as salvage therapy. }\end{array}$ \\
\hline & $\begin{array}{l}\text { 3. Demonstrates very good } \\
\text { efficacy as antifungal prophylaxis } \\
\text { in neutropenic patients. }\end{array}$ & $\begin{array}{l}\text { 3. May be used as a first-line } \\
\text { prophylactic agent in } \\
\text { neutropenic patients. }\end{array}$ \\
\hline & $\begin{array}{l}\text { 4. Demonstrates good efficacy } \\
\text { in the treatment of Candida } \\
\text { esophagitis. }\end{array}$ & $\begin{array}{l}\text { 4. May be used in patients, } \\
\text { refractory to or unable to } \\
\text { tolerate, oral therapy. }\end{array}$ \\
\hline $\begin{array}{l}\text { Patient-oriented } \\
\text { evidence }\end{array}$ & $\begin{array}{l}\text { Multiple randomized clinical trials } \\
\text { show very good outcome data } \\
\text { for prophylaxis in neutropenic } \\
\text { patients and treatment of } \\
\text { candidemia/invasive candidiasis. }\end{array}$ & $\begin{array}{l}\text { Monitoring for potential adverse } \\
\text { effects, especially hepatotoxicity } \\
\text { is necessary while on therapy. }\end{array}$ \\
\hline $\begin{array}{l}\text { Economic } \\
\text { evidence }\end{array}$ & $\begin{array}{l}\text { Acquisition costs are high compared } \\
\text { to oral azoles and conversion to less } \\
\text { expensive oral alternatives should be } \\
\text { accomplished as soon as possible. }\end{array}$ & $\begin{array}{l}\text { Cost-effective for hospitalized } \\
\text { patients with serious infections, } \\
\text { primarily because of efficacy and } \\
\text { decreased length of hospital stay. }\end{array}$ \\
\hline
\end{tabular}

Correspondence: Annette C Reboli Cooper Medical School of Rowan University, 40I South Broadway, Camden, NJ, USA 08I03

$\mathrm{Tel}+\mathrm{I} 85636 \mathrm{I} 2800$

Fax + $85636|280|$

Email reboli@rowan.edu 


\section{Introduction}

Over the last few decades there has been a rise in invasive fungal infections worldwide. These infections, which are associated with significant morbidity and mortality, are often the result of advancements in modern medicine such as hematopoietic stem cell and solid organ transplantation, chemotherapies for autoimmune disorders and malignancies, major surgical procedures, parental nutrition, prosthetic and intravascular devices, and broad spectrum antimicrobial therapies. ${ }^{1}$ The Surveillance and Control of Pathogens of Epidemiological Importance (SCOPE) study analyzed 24,179 cases of nosocomial blood stream infections (BSIs) during the period from March 1995 through September 2002 from 49 hospitals in the United States. The fourth most common BSI was due to Candida species at 4.8 BSIs per 10,000 admissions. Additionally, candidemia was associated with the highest crude mortality rate at $39 \%$. Half of these BSIs were found to occur in intensive care units (ICUs) where the level of acuity is very high and prolonged stays pose a risk for invasive candidiasis. With the aforementioned advancements in modern medicine and an aging population these infections will continue to be a challenging problem. ${ }^{2}$ In another study which retrospectively analyzed a cohort of patients with candidemia, independent risk factors for hospital mortality included prior antibiotics, high Acute Physiology And Chronic Health Evaluation II (APACHE II) scores, and administering antifungal therapy 12 hours after the first blood culture. ${ }^{3}$ A reduced risk of death was independently associated with appropriate empirical antifungal therapy (odds ratio [OR] 0.46; 95\% confidence interval [CI] 0.22-1.00; $P=0.05$ ) in patients with invasive Candida infections during a 5-year period of Canadian population-based surveillance of 207 patients with invasive candidiasis. ${ }^{4}$

Over $90 \%$ of cases of invasive candidiasis are caused by the following species in order of frequency: Candida albicans, Candida glabrata, Candida parapsilosis, Candida tropicalis, and Candida krusei..$^{5}$ In recent years, there has been an increase in non-albicans species. The echinocandins have a broad spectrum of activity against a variety of Candida species including those that are polyene- and azole-resistant. Their safety profile and ease of administration have made them a first choice in treating many Candida infections as outlined in the 2009 Infectious Diseases Society of America (IDSA) guidelines for management of candidiasis. ${ }^{6}$

\section{Methods}

The medical literature was searched for articles published in the English language using the keywords "micafungin," "aspergillosis," "candidemia," "invasive candidiasis," "oral and esophageal candidiasis," "prophylaxis," "pharmacoeconomic analysis," and "clinical trials." The search included articles that were published as of May 2013.

\section{Micafungin}

Micafungin (Astellas Pharma US, Inc, Northbrook, IL, USA) is a semisynthetic lipopeptide synthesized through the chemical modification of a fermentation product of Coleophoma empetri. The molecular formula is $\mathrm{C}_{56} \mathrm{H}_{70} \mathrm{~N}_{9} \mathrm{NaO}_{23} \mathrm{~S}$ with a weight of 1292.26 Daltons. Its complex aromatic side chain, a 3,5 diphenyl-substituted isoxazole ring, differs from the fatty acid side chain of caspofungin and alkoxytriphenyl side chain of anidulafungin and it is soluble in water.,

Like the other echinocandins, micafungin is a concentration dependent noncompetitive inhibitor of 1,3- $\beta$-D-glucan synthase. This enzyme complex, which is involved in the synthesis of the glucan polymers of the fungal cell wall, is encoded by the genes $F K S 1$ and $F K S 2 .{ }^{9}$ A disruption in the synthesis of these polymers leads to osmotic instability and eventual cell death. Mammalian cells do not contain glucan and thus are not susceptible to this toxicity. This unique mechanism of action may also prevent cross-resistance with other antifungals that interfere with DNA synthesis or act at the level of the cell membrane. ${ }^{10}$ Resistance to echinocandins has been relatively infrequent to date. Mutations in two highly conserved regions ("hot spots") of the $F K S$ genes are responsible for decreased susceptibility to the echinocandins. ${ }^{11}$ Clinical breakpoints for Candida species have been revised by the Clinical and Laboratory Standards Institute (CLSI) for the echinocandins. This revision, which is species specific, is more sensitive to detect mutational or acquired resistance mechanisms and hence predict clinical outcomes. ${ }^{12}$

Micafungin pharmacokinetics have been studied in healthy adult human volunteers, adult volunteers with renal dysfunction and those with moderate hepatic disease, as well as, ill and hospitalized children and adults. It is metabolized by arylsulfatase with secondary metabolism by catechol-Omethyltransferase. Although it is a substrate for, and a weak inhibitor of, cytochrome P450 3A4 in vitro, hydroxylation by this pathway is not significant in vivo. Micafungin is not a substrate or inhibitor of P-glycoprotein. Protein binding is more than $99.5 \%$, and $90 \%$ of the parent drug and its metabolites are excreted in the bile. Less than $1 \%$ is excreted unchanged in the urine. It does not need adjustment for renal or hepatic dysfunction and it is not dialyzable. ${ }^{8,13}$

Following intravenous administration, micafungin demonstrates dose-proportional linear pharmacokinetics. It has 
a low clearance of $0.197 \mathrm{~mL} / \mathrm{minute} / \mathrm{kg}$ and the elimination half-life is 13.9 hours over the dose range of 12.5 to $150 \mathrm{mg}$ daily. ${ }^{14}$ In children, there is an inverse relationship between clearance and age where those of 2 to 8 years of age demonstrated a clearance 1.35 times that of patients aged 9 years or older. ${ }^{15}$ Of interest, the systemic clearance in obese patients increases with increases in weight, which suggests that dosing levels may need to be individualized. ${ }^{16}$

In a study of lung transplant recipients, a single dose of $150 \mathrm{mg}$ resulted in concentrations that exceeded the minimum inhibitory concentration required to inhibit the growth of $90 \%$ of organisms $\left(\mathrm{MIC}_{90}\right)$ of Aspergillus fumigatus in epithelial lining fluid, alveolar cells, and plasma during the entire 24 hour dosing period. Additionally, multiple-dose simulations demonstrated that these concentrations would continue to rise during a course of 14 days. ${ }^{17}$ Neither a single dose nor multiple doses of micafungin administered with cyclosporine, tacrolimus, mycophenolate, prednisolone, fluconazole, or voriconazole had an effect on their pharmacokinetics. Itraconazole, sirolimus, and nifedipine dosing should be reduced when administered with micafungin. ${ }^{7}$ Micafungin should be infused over 1 hour in order to avoid histamine mediated reactions. It is a pregnancy category $\mathrm{C}$ agent and it is not known if it is excreted in human breast milk. ${ }^{7}$

Device-related Candida infections are difficult to treat because many antimicrobials are unable to penetrate the complex biofilms (sessile fungi, a hyphal layer, and an extracellular matrix) that form on these devices. The echinocandins have been shown to have unique activity against the biofilms associated with various Candida species. In an in vitro model of a C. albicans, biofilm associated with silicone catheters, caspofungin $(2 \mathrm{mg} / \mathrm{L})$, and micafungin $(5 \mathrm{mg} / \mathrm{L})$ used as lock solutions were able to reduce the metabolic activity of intermediate and mature biofilms and may therefore be candidates for the reduction or control of candida biofilms on silicone medical devices when used as part of an antifungal lock. ${ }^{18}$ In another study, clinical isolates of C. albicans were used to determine the planktonic and sessile susceptibilities of micafungin where the $\mathrm{MIC}_{90} \mathrm{~s}$ were $0.125 \mu \mathrm{g} / \mathrm{mL}$ and $1.0 \mu \mathrm{g} / \mathrm{mL}$, respectively. ${ }^{19}$ More recently, the activity of all three echinocandins (caspofungin, micafungin, and anidulafungin) against planktonic cells and biofilms from BSIs of various Candida species was studied. While planktonic cells of all Candida species were susceptible to all three compounds, this was not the case for the biofilms, where there were drug and species specific differences. The MICs of all three echinocandins for C. albicans and C. krusei biofilms were $\leq 1 \mathrm{mg} / \mathrm{L}$, but $\geq 32 \mathrm{mg} / \mathrm{L}$ for Candida guilliermondii and Candida lusitaniae. All echinocandins had high MICs against $C$. parapsilosis, but micafungin had the lowest at $4 \mathrm{mg} / \mathrm{L}$ (anidulafungin was $32 \mathrm{mg} / \mathrm{L}$ and caspofungin was $64 \mathrm{mg} / \mathrm{L}) .^{20}$

\section{Clinical efficacy Prophylaxis}

The risk for fungal infections such as Candida and Aspergillus in patients undergoing hematopoietic stem cell transplantation (HSCT) is associated with the degree and duration of neutropenia, the use of corticosteroids, and graft versus host disease. ${ }^{21}$ Two multicenter trials of adults undergoing HSCT were able to demonstrate that fluconazole reduced the frequency of invasive candidiasis when compared with placebo. ${ }^{22,23}$ Although this was an important advancement, patients still faced the risk of infection with fluconazoleresistant Candida and Aspergillus. The echinocandins with their favorable safety profile, once daily dosing, and spectrum of activity that includes Aspergillus in addition to Candida make them attractive for use in this patient population.

With the hypothesis that the echinocandin, micafungin, would be an effective antifungal prophylaxis in HSCT patients during periods of neutropenia, a prospective, randomized, double-blind, multi-institutional, comparative Phase III trial of micafungin and fluconazole was designed and conducted to test this hypothesis. ${ }^{24}$ Patients were eligible for participation if they were to receive an autologous HSCT for a hematologic malignancy or an allogeneic HSCT for any indication. Participants could not have received an antifungal drug within 72 hours of the first dose of the study drug, had to be free of invasive fungal infections at the time of enrollment, and without liver disease, defined as serum aminotranferases greater than five times the upper limit of normal or bilirubin greater than 2.5 times the upper limit of normal, and had to be at least 6 months of age. Patients were randomized to either micafungin or fluconazole using a 1:1 schedule. They were stratified by type of transplant (autologous, matched sibling allograft, matched unrelated donor), age (6 months to 12 years and $\geq 13$ years), and study center. Allogeneic transplant recipients were further stratified according to transplant mortality. ${ }^{24}$ Randomized treatment was administered during the pre-engraftment or neutropenic phase of HSCT with either $50 \mathrm{mg}$ of micafungin $(1 \mathrm{mg} / \mathrm{kg}$ in those weighing $<50 \mathrm{~kg})$ or $400 \mathrm{mg}$ of fluconazole $(8 \mathrm{mg} / \mathrm{kg}$ in those weighing $<50 \mathrm{~kg}$ ) over 1 hour once daily. The duration of the study drug continued until the earliest of the following: suspected, probable or proven invasive fungal infection; $\leq 5$ days after engraftment (absolute neutrophil count of $\geq 500$ cells $/ \mathrm{mm}^{3}$ following the 
nadir absolute count); treatment day 42 after HSCT; unacceptable drug toxicity; patient withdrawing from study or investigator stopping the study drug; or death. ${ }^{24}$

Treatment success, the primary end point, was defined as no suspected, probable, or proven systemic fungal infection through the end of prophylaxis therapy and no probable or proven fungal infection through the end of the 4-week post treatment period. The analysis of secondary efficacy variables included the frequency of probable or proven fungal infections throughout the post-treatment period, the administration of antifungal drugs for suspected fungal infections, and the pathogen-based frequency of proven infections. Additional secondary outcomes that were measured included the frequency of fungal colonization and superficial fungal infection, the time to suspected fungal infection, the time to treatment failure, and mortality. Safety analyses were also performed. ${ }^{24}$

A total of 1267 patients were screened for eligibility and 889 were enrolled. Those that received at least one dose of the study drug at the beginning of the neutropenic or preengraftment phase included 425 in the micafungin arm and 457 in the fluconazole arm. These patients provided data for the primary end point in the modified intent-to-treat (mITT) analysis. The demographic characteristics of both treatment groups were similarly balanced. ${ }^{24}$

Patients in the micafungin arm had a higher overall treatment success rate at $80.0 \%$ versus $73.5 \%$ in the fluconazole arm (absolute difference, $+6.5 \%$; 95\% CI, 0.9\%-12\%; $P=0.03)$. Six breakthrough Candida infections occurred as BSIs. In the micafungin arm, three of these infections were during the prophylactic period and were due to C. albicans, C. parapsilosis, and C. lusitaniae, and the fourth was due to $C$. glabrata during the post-treatment follow-up period. Two episodes of candidemia occurred in the fluconazole arm during prophylactic treatment and were due to C. parapsilosis and $C$. krusei. One probable breakthrough aspergillosis occurred in the micafungin arm and three in the fluconazole arm where an additional four proven cases occurred $(P=0.071)$. Fusariosis occurred in one patient on micafungin and two on fluconazole. One case of zygomycosis occurred in the micafungin arm. Empirical antifungal therapy was administered to $15.1 \%$ of patients in the micafungin arm and $21.4 \%$ in the fluconazole arm $(P=0.024) .{ }^{24}$

A total of 44 deaths occurred during the study and none were attributed to the study drug. One of the 18 deaths in the micafungin arm was from zygomycosis and two deaths in the fluconazole arm were from pulmonary aspergillosis. Adverse events that resulted in discontinuation of the study drug occurred less frequently among those on micafungin than those on fluconazole, but was not statistically significant (4.2\% versus $7.2 \%, P=0.58)$. The frequency of adverse events was similar between pediatric and adult patients. ${ }^{24}$ This randomized, double-blind, comparative Phase III trial demonstrated the efficacy of micafungin for antifungal prophylaxis in neutropenic patients.

Patients with hematologic malignancies with continuing febrile neutropenia from chemotherapy require the addition of empiric antifungal therapy to broad spectrum antibiotics. ${ }^{25}$ Like HSCT patients, the resulting neutropenia in this population carries a risk of $2 \%-40 \%$ for invasive fungal infection. ${ }^{26}$ In a prospective non-randomized study to evaluate the efficacy and safety of micafungin for empiric therapy in patients with hematologic malignancies with febrile neutropenia, 23 patients with recurrent or persistent fever after at least 3-5 days of antibacterial therapy were enrolled. ${ }^{27}$ Patients received between $50 \mathrm{mg}$ and $300 \mathrm{mg}$ of micafungin once daily for a minimum of 5 days until the absolute neutrophil count was greater than $500 / \mu \mathrm{L}$ and temperature was $<37^{\circ} \mathrm{C}$ for more than 2 consecutive days. Patients were excluded if they were pregnant or lactating, HIV positive, had an allergy to the study drug, or received antifungal therapy within 72 hours before registration. Treatment success, the primary efficacy endpoint, was defined as the resolution of fever during the neutropenic period and cure of a fungal infection if it was present at baseline. ${ }^{27}$

The following antifungal drugs were administered as prophylaxis to $95.6 \%$ of patients: oral fluconazole $39.1 \%$, intravenous fluconazole $21.7 \%$, oral itraconazole $26 \%$, intravenous fluconazole with itraconazole and amphotericin B $4.4 \%$, and intravenous fluconazole with amphotericin B $4.4 \%$. The median duration of neutropenia was 14 days (5-43 days) and the median duration of treatment was $17.8 \pm 9.9$ (5-43 days). Although four patients (19.1\%) had a positive $\beta$-D-glucan assay before the initiation of treatment, none had fungus detected in blood cultures. Eight patients (38.1\%) had infiltrates on baseline radiographs. The majority of patients $(43.5 \%)$ received the daily maximum dose of $150 \mathrm{mg} .{ }^{27}$ Treatment success was seen in 17 patients $(73.9 \%)$. Although six patients experienced treatment failure because of lack of improvement in clinical symptoms, none had breakthrough fungal infections. The degree of neutropenia did not influence the success rate and the daily dose of micafungin of $100 \mathrm{mg}$ or more tended to be effective. The response rate in those with abnormal radiographs at baseline was $75 \%$ and all those with positive $\beta$-D-glucan assays prior to the study drug had negative assays after receiving micafungin. 
Adverse events, which occurred in five patients (21.7\%), were mild or moderate abnormal liver enzymes, that did not require discontinuation of the study drug, and all resolved after finishing micafungin. ${ }^{27}$ Although the number of patients in this study was small, monotherapy with micafungin for febrile neutropenia in patients with hematologic malignancies appeared to be safe and efficacious.

\section{Empirical therapy}

Fifty-three patients, between the ages of 26-92 years were enrolled in a prospective, consecutive entry study to evaluate the effect and safety of empirical therapy with micafungin in patients with hematological malignancies with febrile neutropenia who were unresponsive to antibacterial therapy. ${ }^{28}$ Antifungal prophylaxis in the form of oral fluconazole was permitted but discontinued at the time of starting the study drug. As per the guidelines of the Infectious Diseases Society of America, antibacterial therapy was started at the beginning of febrile neutropenia. ${ }^{25}$ If febrile neutropenia continued for 72 hours, then micafungin was added at a daily dose of $150 \mathrm{mg}$ which could be increased to $300 \mathrm{mg}$ if the response was not appropriate. Micafungin was continued until resolution of fever and for up to 3 days after neutrophil recovery. Granulocyte-colony stimulating factor was permitted. ${ }^{28}$

Treatment success, the primary endpoint, occurred if there were no breakthrough fungal infections, the patient survived 7 days after the study drug was discontinued, fever resolved during neutropenia, a baseline fungal infection resolved, and the study drug was not prematurely discontinued. The overall efficacy of micafungin was $70 \%$. All patients who were positive for $\beta$-D-glucan or galactomannan were negative after starting micafungin. Two patients died from breakthrough fungal infections. One had a positive galactomannan antigen and radiographic findings suggestive of probable pulmonary aspergillosis and the other had proven invasive pulmonary aspergillosis at autopsy. Adverse reactions, which occurred in six patients, were due to hepatic dysfunction and did not require discontinuation of the study drug. The median plasma levels of micafungin were studied in 15 patients at 1 and 4 hours after administration and were $15.15 \mu \mathrm{g} / \mathrm{mL}$ (range $8.53-29.48 \mu \mathrm{g} / \mathrm{mL}$ ) and $9.96 \mu \mathrm{g} / \mathrm{mL}$ (range $5.25-18.67 \mu \mathrm{g} / \mathrm{mL}$ ), respectively, and the trough was $4.85 \mu \mathrm{g} / \mathrm{mL}$ (range $1.49-19.03 \mu \mathrm{g} / \mathrm{mL}$ ). These levels were reported as remaining above the MICs for most fungal pathogens for 24 hours. Additionally, hepatic or renal dysfunction did not correlate with the dose or plasma level of micafungin. ${ }^{28}$ Hence, this study demonstrated micafungin to be safe and efficacious when used as empiric antifungal therapy in patients with hematologic malignancies with febrile neutropenia.

A Phase I, open-label, sequential-group dose escalation study was conducted to assess the tolerability, safety, and pharmacokinetics of micafungin in pediatric patients with neutropenia. Within 24 hours of starting broad spectrum antibacterial agents for new onset febrile neutropenia, micafungin was initiated at $0.5 \mathrm{mg} / \mathrm{kg} /$ day and escalated to a maximum of $4 \mathrm{mg} / \mathrm{kg} /$ day. Seventy-seven patients were stratified by ages 2 to 12 years and 13 to 17 years. A maximum tolerated dose was not identified and there were no dose-related adverse events. Adverse events that did occur were considered mild to moderate and the one death that occurred during the study was due to septic shock. Linear pharmacokinetics and an inverse relationship between clearance and age were observed. As in adults, dose-proportional linear pharmacokinetics were observed in children. The higher clearance in younger patients suggests an increased dose may be needed in the very young. Further studies are needed to determine the optimal dose of micafungin for the prevention and treatment of invasive fungal infections in pediatric patients. ${ }^{15}$

\section{Candida esophagitis}

Esophageal candidiasis with or without oropharyngeal involvement occurs in patients with immunodeficiencies. The resulting odynophagia, dysphagia, ulcers, and erosions are responsible for significant morbidity. It is often seen in patients with advanced human immunodeficiency virus (HIV) where the mean incidence has been reported at $10 \%-15 \%{ }^{29}$

An open-label study to assess the efficacy and safety of micafungin in HIV positive patients with esophageal candidiasis confirmed and graded by endoscopy was conducted in nine centers in South Africa. In order to determine the minimum effective dose of micafungin that would produce a clinical improvement or cure in $65 \%$ of patients after 10 days of study drug, patients received $12.5 \mathrm{mg}, 25 \mathrm{mg}$, $50 \mathrm{mg}, 75 \mathrm{mg}$, or $100 \mathrm{mg}$ of daily micafungin. The duration of treatment was 14 days but could be extended to 21 days and there was a 2-week follow-up. The primary efficacy endpoint was improvement or cure of clinical symptoms and signs. Secondary efficacy endpoints included mycological response at the end of therapy (EOT), improvement in mucosal lesions, the incidence of relapse at 2 weeks following the EOT, improvement in baseline clinical and endoscopic grades at the EOT, and no discontinuation of the study drug because of lack of efficacy or adverse events. ${ }^{30}$ 
A full analysis set included 120 patients who were enrolled and received at least one dose of micafungin. After 36 patients were excluded, 84 patients remained in the per protocol set. No significant demographic differences were seen in the five dose groups who had a median CD4 of $27 \mathrm{cells} / \mathrm{mm}^{3}$. Patients in the per protocol set received a mean duration of $15.1 \pm 3.72$ days (range 7-23 days) of study drug. All dose groups demonstrated a clinical response with the exception of one patient in the $12.5 \mathrm{mg}$ dose group, but there was a doserelated response rate in the number of patients who experienced clearing of clinical signs and symptoms $(100 \%$ who received 75 or $100 \mathrm{mg}, 95 \%$ who received 25 and $50 \mathrm{mg}$, and $67 \%$ who received $12.5 \mathrm{mg}, P=0.001$ ). This response was not influenced by baseline esophageal candidiasis severity or CD4 count. In those who received $\geq 75 \mathrm{mg}$, an endoscopic grade of 0 was achieved. Because all doses met the criteria for clinical efficacy, the true minimum effective dose was not identified, but was suggested to be $12.5 \mathrm{mg} .{ }^{30}$

Ninety patients (75.5\%) experienced at least one adverse event and the majority of these events were considered mild to moderate. Only one patient had an adverse event (diarrhea) that was considered to be serious and drug-related. The most common events were vomiting (6.7\%), abnormal liver function tests (5.8\%), nausea (5.0\%), and rash (3.3\%). Two patients discontinued micafungin because of an adverse event (increased liver function tests and presumed erythema multiforme). There were no infusion- or histamine-related reactions or nephrotoxicity. The 13 deaths that occurred during the study were attributed to acquired immunodeficiency syndrome (AIDS) related events and not the study drug. ${ }^{30}$ This study showed micafungin to be well-tolerated and safe in patients with documented esophageal candidiasis. There was a dose-response relationship suggested by the number of patients who experienced resolutions of signs and symptoms of esophagitis.

Fluconazole has been the preferred treatment for esophageal candidiasis in HIV positive patients, but increasing azole-resistant strains have emerged. ${ }^{29,31}$ In order to determine the optimal dose of micafungin for the treatment of esophageal candidiasis in HIV positive patients, a randomized, double-blind, parallel-group study was compared with fluconazole. ${ }^{32}$

Two hundred and fifty-one HIV positive patients, 18 years of age or older, with clinical symptoms of esophageal candidiasis confirmed by endoscopy and culture were included. Randomization was 1:1:1:1 to receive daily micafungin at 50,100 , or $150 \mathrm{mg}$, or fluconazole at $200 \mathrm{mg}$. The duration of treatment was 14 days which could be extended to 21 days in order to achieve endoscopic clearance. An endoscopic cure rate at the EOT was the defined primary efficacy end point. Secondary efficacy assessments included the endoscopic cure rate at day 14 , changes in baseline endoscopic findings compared with those of the cure rate, the esophageal candidiasis severity score, clinical response and overall therapeutic success at the end of treatment, and the incidence of relapse. Susceptibility testing was performed on baseline isolates as well as those at the end of treatment. ${ }^{32}$

The intent to treat (ITT) population included 245 patients who received micafungin $50 \mathrm{mg}, 100 \mathrm{mg}$, or $150 \mathrm{mg}$, or fluconazole $200 \mathrm{mg}$ daily. Demographic characteristics were similar between the groups. The per protocol population included 199 patients. Discontinuation rates of micafungin and fluconazole were similar. The endoscopic cure rate was dose-related for the ITT population at $68.8 \%, 77.4 \%$, and $89.9 \%$ for micafungin doses 50,100 , and $150 \mathrm{mg}$, respectively. When differences in endoscopic cure rates for the three micafungin groups were compared, statistical significance was observed. The per protocol population results confirmed the findings in the ITT population. When comparing endoscopic cure rates for micafungin $100 \mathrm{mg}$ versus fluconazole or micafungin $150 \mathrm{mg}$ with fluconazole, there was no difference statistically. In the 138 patients with a baseline endoscopic grade 3, a stratified analysis showed fluconazole to have a lower endoscopic cure rate at $77.0 \%$ versus $100 \%$ achieved by micafungin $150 \mathrm{mg} .{ }^{32}$

The rates of mycological eradication for micafungin $50 \mathrm{mg}, 100 \mathrm{mg}$, and $150 \mathrm{mg}$ were $35.1 \%, 78.3 \%$, and $57.1 \%$, respectively, and $67.3 \%$ for fluconazole. Endoscopic findings, from baseline to EOT, improved in all treatment groups and were dose-related in the micafungin groups. Micafungin dose-dependent improvements were also seen on day 14 and the EOT. Therapeutic success was $79.7 \%, 83.9 \%$, and $91.5 \%$ in the $50 \mathrm{mg}, 100 \mathrm{mg}$, and $150 \mathrm{mg}$ micafungin groups, respectively, versus $91.5 \%$ in the fluconazole group. There were nine patients who relapsed in the micafungin group and none in the fluconazole group during the posttreatment period. Although these nine patients achieved an EOT endoscopic grade 0, they received "nonprophylactic therapy" and/or had a worsening of symptoms during the 2-week follow-up period. Only one of these reverted back to their baseline clinical symptoms. It was unclear if they were experiencing a relapse or reinfection. The majority of all causality adverse events were rated as mild to moderate and occurred in $93.3 \%$ of those in the micafungin group and $89.2 \%$ of those in the fluconazole group. The most common were fever, headache, nausea, diarrhea, abdominal pain, 
injection-site inflammation, and leukopenia. Mild elevations in liver function tests occurred in $12.7 \%$ with no difference between the treatment groups. Renal related events were seen in $3.8 \%$ of those on micafungin and none on fluconazole. No patients discontinued either drug because of renal or hepatic adverse events. There were no differences in the tolerability and safety of either drug, and the ten deaths that occurred during the post treatment period were not related to either study drug. Therefore, micafungin at a dose of $100 \mathrm{mg}$ or $150 \mathrm{mg}$ was comparable to $200 \mathrm{mg}$ of daily fluconazole with respect to efficacy, safety, and tolerability. ${ }^{32}$

A multicenter, multinational, randomized, double-blind, parallel-group, non-inferiority study comparing intravenous micafungin to intravenous fluconazole for the treatment of esophageal candidiasis was performed in 523 patients aged 16 years or older with clinical symptoms of esophageal candidiasis confirmed by endoscopy. Patients received daily micafungin at $150 \mathrm{mg}$ or fluconazole at $200 \mathrm{mg}$ for a minimum of 14 days or for 7 days following resolution of clinical symptoms of esophageal candidiasis, whichever was longer. The endoscopic cure rate defined as a grade 0 at the EOT was the primary efficacy endpoint. Secondary clinical efficacy assessments included EOT mucosal and clinical response as well as the overall therapeutic response, the incidence of post-treatment relapse, and changes in clinical symptoms and endoscopic finding of esophageal candidiasis at the EOT compared with baseline. ${ }^{33}$

The primary analysis set was comprised of 518 patients who received at least one dose of study drug. The baseline demographics as well as the severity of mucosal lesions and signs and symptoms of esophageal candidiasis were similar between the two groups. The majority of patients had confirmed or suspected HIV where the baseline CD4 was $109.0 \pm 190.80$ cells $/ \mathrm{mL}$ in the micafungin group and $105.9 \pm 176.72$ cells $/ \mathrm{mL}$ in the fluconazole group. Only a few HIV positive patients received antiretroviral therapy $(8.5 \%$ on micafungin and $11.6 \%$ on fluconazole) and approximately $14 \%$ in each group had tuberculosis. ${ }^{33}$

The median duration of therapy for both groups was 14 days and the endoscopic cure rate was $87.7 \%$ for those who received micafungin versus $88.0 \%$ for those who received fluconazole. The clinical and overall therapeutic success rates were $94.2 \%$ and $87.3 \%$, respectively, for micafungin and $94.6 \%$ and $87.2 \%$, respectively, for fluconazole. The overall relapse rate was similar between the groups at $15.2 \%$ for micafungin versus $11.3 \%$ for fluconazole. At least one adverse event occurred in $27.7 \%$ of the patients on micafungin and in $21.3 \%$ on fluconazole. A total of
58 patients died during the study and all but one death were not considered to be related to the study drugs. Adverse events that led to the discontinuation of micafungin in six patients included rash, delirium, and progression of AIDS; and delirium and asthenia in two patients on fluconazole. Mean levels of liver function tests were similar between the groups at the EOT where only one patient from each group had transaminase levels greater than three times the upper limit of normal. This study demonstrated that micafungin at a daily dose of $150 \mathrm{mg}$ was as efficacious as fluconazole $200 \mathrm{mg}$ daily. ${ }^{33}$

\section{Invasive aspergillosis}

Patients with significant immunosuppression are at risk for invasive aspergillosis which is associated with high mortality. The echinocandins, which have been studied predominately as salvage therapy, are an alternative therapy for invasive pulmonary aspergillosis as outlined in the 2008 Clinical Practice Guidelines of the Infectious Diseases Society of America. The guidelines also recommend them as an alternative therapy for cutaneous lesions secondary to disseminated disease. ${ }^{34}$ The safety and efficacy of micafungin were evaluated in a multinational, non-comparative study in probable (pulmonary only, where either radiographic findings were consistent with the diagnosis or there was a positive respiratory culture) or proven (tissue from any infected site with hyphae with or without a confirmatory culture or a sterile site positive culture) Aspergillus species infection in a diverse patient population. This open-label study enrolled pediatric and adult patients (ages 3 months to 82 years of age) with acute invasive aspergillosis who were unable to tolerate other therapy and labeled as "toxicity failure," did not respond to or failed prior therapy and were labeled as "refractory," or received $\leq 48$ hours of therapy and were labeled as "primary." The vast majority of patients had pulmonary infection. Only one patient had central nervous system/brain involvement. Micafungin was initiated at $75 \mathrm{mg} /$ day or $1.5 \mathrm{mg} / \mathrm{kg} /$ day for patients weighing $\leq 40 \mathrm{~kg}$ but could be increased after 7 days if the patient was tolerating therapy but not improving clinically or microbiologically or if there was progression of disease. This escalation in dosing was done at increments of $75 \mathrm{mg} /$ day or $1.5 \mathrm{mg} / \mathrm{kg} /$ day for patients weighing $\leq 40 \mathrm{~kg}$ to a maximum of $200 \mathrm{mg} /$ day per the European protocol. The non-European protocol permitted doses above $225 \mathrm{mg} /$ day or $4.5 \mathrm{mg} / \mathrm{kg}$ /day for patients weighing $\leq 40 \mathrm{~kg}$ at the discretion of the medical monitor. The duration of micafungin was at least 7 days to a maximum of 90 days. Post-treatment follow-up was 6 weeks. In the 
toxicity failure group, previous antifungal therapy had to be discontinued before initiating micafungin and those in the refractory group could receive micafungin monotherapy or have it added to their current therapy. ${ }^{35}$

Treatment success based on the global assessment at the EOT was defined as the primary efficacy endpoint. Based on clinical, microbiological, and radiographic findings, responses were labeled as partial or complete, stabilization of disease, or failure. Patients who received at least one dose of micafungin comprised the full analysis set (FAS) and were included in the safety analysis. The per protocol set included those who received at least seven doses of micafungin for probable or proven invasive aspergillosis at baseline. Patients in the FAS who met the criteria as specified by the protocol were analyzed as the modified FAS (mFAS). ${ }^{35}$

Multiple international centers enrolled 331 patients, of whom 326 comprised the FAS and 204 were in the per protocol set. A total of 101 patients in the FAS were excluded because they did not meet the per protocol diagnosis; the remaining 225 in the FAS comprised the mFAS. Within this latter group, four patients were enrolled as toxicity failures, 29 as primary, and 192 as refractory. Combination therapy was administered to $191 / 225$ patients in the mFAS and the majority of these were in the refractory group. Of the 192 in the refractory group, 174 received combination therapy. A lipid preparation of amphotericin B was administered to $148 / 192$ patients at a mean daily dose of $6.09 \mathrm{mg} / \mathrm{kg}$ for a mean duration of 23 days. Eighty-six of the 192 patients had received amphotericin B deoxycholate. Itraconazole was administered to $66 / 192$ patients for a mean of 47 days. Five patients received prior voriconazole, seven received caspofungin, and five received posaconazole. The mean daily dose of micafungin in adults (FAS) was $111.4 \pm 50.97 \mathrm{mg}(1.7 \pm 0.82 \mathrm{mg} / \mathrm{kg} /$ day $)$ for a mean duration of $53.6 \pm 50.95$ days and $67 \%$ received at least one dose escalation. For pediatric patients less than 16 years of age, the mean daily dose was $2.1 \pm 1.25 \mathrm{mg} / \mathrm{kg}$. ${ }^{35}$

A favorable response was seen in $80 / 225$ (35.6\%) of patients in the mFAS group, $11.1 \%$ had stabilization of their disease, and $53.3 \%$ had progression of their disease. For the 34 patients who received micafungin monotherapy, $15(44.4 \%)$ had a favorable response. In those who did not have a dose escalation above the $75 \mathrm{mg} / \mathrm{kg} /$ day, 30/96 (31.1\%) had a favorable response. Patients who had neutrophil recovery had a more favorable response than those who did not ( $18 / 36$ or $50 \%$ versus $5 / 30$ or $16.7 \%$ ). A favorable response rate of $25.9 \%$ was seen in all allogeneic HSCT, $49.4 \%$ in leukemic or solid tumor patients undergoing chemotherapy, and $44.8 \%$ in children overall. Patients with HIV/AIDS had a low response rate of $16.7 \%$. Adverse events judged to be possibly or probably related to the study drug occurred in 104/326 (31.9\%) patients and treatment was stopped in 85 of these patients, of whom 17/70 were children. There were a total of 183 deaths during therapy and in the 6-week follow-up among the 326 patients enrolled, of which 107 were considered to be because of invasive aspergillosis. At the 6-week follow-up visit, 47 of the 145 patients in the mFAS had a partial or complete response. These response rates for micafungin compare favorably with other antifungal agents. Micafungin alone or in combination with amphotericin B was generally well-tolerated and is another option for the treatment of invasive aspergillosis. ${ }^{35}$

In a further analysis of the $98 \mathrm{HSCT}$ recipients (88 allogeneic and ten autologous) from the above study, eight received micafungin monotherapy and 90 received micafungin with another antifungal. The majority (73) received amphotericin $\mathrm{B}$ with micafungin, ten received a combination of amphotericin B plus itraconazole, two received amphotericin plus fluconazole, one received itraconazole, one received itraconazole plus fluconazole, and one received amphotericin plus voriconazole. The mean dose of micafungin was $105 \pm 60 \mathrm{mg}$ /day and the mean duration of treatment was $51 \pm 60$ days. A partial plus complete or overall response rate occurred in 25/98 (26\%) patients and 12 patients had stable disease. The response to treatment was not influenced by the type of HSCT, Aspergillus species, or site of infection. Micafungin was well-tolerated with little toxicity or adverse events. In this high-risk patient population, micafungin alone or in combination with other antifungal agents is a reasonable option for treatment of invasive aspergillosis. ${ }^{36}$

\section{Candidemia and other forms of invasive candidiasis}

The US Food and Drug Administration has approved micafungin for the treatment of candidemia, acute disseminated candidiasis, Candida peritonitis and intra-abdominal abscesses, esophageal candidiasis, and prophylaxis of Candida infections in patients undergoing $\mathrm{HSCT}^{7}$ In the European Union, micafungin is approved for the treatment of patients with invasive candidiasis, esophageal candidiasis in patients for whom intravenous therapy is appropriate, and prophylaxis of Candida infection in patients undergoing HSCT or in patients who are expected to have neutropenia for 10 or more days. ${ }^{37}$ The 2009 IDSA Clinical Practice Guidelines for the Management of Candidiasis have placed an emphasis on 
fluconazole and echinocandins as the preferred choices for proven and suspected invasive candidiasis. ${ }^{6}$ Little distinction is made clinically between the three available echinocandins. An echinocandin is favored for non-neutropenic patients with moderately severe to severe illness or among patients who have had recent azole exposure for treatment or prophylaxis. Among patients with proven or suspected infection due to C. glabrata, an echinocandin is preferred as initial therapy. For neutropenic patients, an echinocandin is favored for most patients if the Candida species is unknown. Treatment for other forms of invasive candidiasis generally follows the recommendations for the treatment of candidemia. Because of poor penetration into cerebrospinal fluid, echinocandins should not be used to treat meningitis. In a recent individual patient-level quantitative review of randomized trials for invasive candidiasis, echinocandin therapy was associated with treatment success and improved survival, compared with other antifungal agents. ${ }^{38}$ The IDSA treatment guidelines are in the process of being updated.

The efficacy of micafungin for the treatment of serious candidal infections has been studied in both adult and pediatric patients (including neonates) in large, randomized, double-blind, multinational, non-inferiority clinical trials. In a Phase III randomized, double-blind study of adult patients, micafungin at $100 \mathrm{mg}$ intravenously daily was compared to liposomal amphotericin B (LAmB) at $3 \mathrm{mg} / \mathrm{kg} /$ day for the treatment of candidemia and other forms of invasive candidiasis. ${ }^{39}$ Eighty-five percent of patients had candidemia. Dosage adjustments, based on efficacy, tolerability, and/or body weight, were permitted in this trial and allowed for the micafungin dose to be increased to $200 \mathrm{mg}$ daily and the $\mathrm{LAmB}$ dose to be increased to $5 \mathrm{mg} / \mathrm{kg} /$ day. The dose of LAmB could be decreased by $50 \%$ if there was nephrotoxicity. The primary efficacy endpoint was the clinical response and the mycological response at the end of intravenous therapy in the per protocol population. In this study, it was required that the conclusion of non-inferiority, defined as the lower bound of the two-sided 95\% confidence interval for the between-group difference exceed $-15 \%$, in not only the per protocol population, but also in the ITT and mITT populations. Of 202 patients in the micafungin arm, overall treatment success was seen in 181 (89.6\%). Comparable results were seen in the LAmB arm with overall success seen in 170 of 190 patients (89.5\%). C. albicans infections were the most common (40\%). The overall success rate by species was comparable between the two groups. Success rates by species for the micafungin arm were as follows: C. albicans $88.4 \%$, C. tropicalis $92.3 \%$, C. parapsilosis $89.2 \%$, and C. glabrata
$82.6 \%$. Micafungin was also non-inferior to $\mathrm{LAmB}$ in a variety of secondary analyses, including neutropenic status at baseline, APACHE II score at baseline, whether subjects had candidemia or other forms of invasive candidiasis, and catheter management. Relapse was rare $(<1 \%)$. ${ }^{39}$

A post hoc analysis of this study examined the subset of patients who were in the ICU and found no significant difference in the overall success rate between the micafungin and LAmB arms (62.5\% versus 66.4\%). For those who were not in the ICU, however, the overall success rate was significantly higher in the micafungin group $(85 \%)$ versus the LAmB group $(72.1 \%)(P=0.01)$. When ICU patients were compared with non-ICU patients, the overall treatment success rate in ICU patients was $64.3 \%$ versus $78.3 \%$ in non-ICU patients $(P=0.0006)$. This difference was found to reflect the baseline APACHE II score. ${ }^{40}$

In the largest randomized, double-blind study of treatment for candidemia and invasive candidiasis, micafungin at two doses, $100 \mathrm{mg}(\mathrm{n}=191)$ and $150 \mathrm{mg}(\mathrm{n}=199)$ was compared to caspofungin at $50 \mathrm{mg}(\mathrm{n}=188) .{ }^{41}$ This study is the first comparison of echinocandins. Success at the end of intravenous therapy was comparable among the groups and numerically better, although not statistically better, for micafungin at $100 \mathrm{mg}$ intravenously daily. Patients could switch to oral fluconazole $400 \mathrm{mg}$ daily after at least 10 days of intravenous therapy. The primary efficacy endpoint was overall treatment success (both clinical and mycological) in the mITT population. Overall success rates were $76.4 \%$, $71.4 \%$, and $72.3 \%$ for micafungin $100 \mathrm{mg}$ daily, micafungin $150 \mathrm{mg}$ daily, and caspofungin $50 \mathrm{mg}$ daily, respectively. The micafungin arms remained non-inferior to caspofungin at 2 and 6 weeks after the EOT. There was no significant difference between treatment groups for clinical success or mycological success individually. In addition, success rates were similar irrespective of neutropenic status at baseline, APACHE II scores at baseline, Candida species, whether the patient had candidemia or other forms of invasive candidiasis, or catheter management. There was a statistically significant difference in the prevalence of $C$. parapsilosis and a numerical difference that was not statistically significant in the prevalence of $C$. glabrata among the treatment groups at baseline. For patients with infections caused by C. parapsilosis, C. glabrata, or C. tropicalis, there were subtle differences in outcome that were not statistically significant. Persistently positive cultures were seen in $5.8 \%$ of patients receiving micafungin $100 \mathrm{mg}$ daily, $11.6 \%$ of patients receiving micafungin $150 \mathrm{mg}$ daily, and $9.6 \%$ of patients receiving caspofungin $50 \mathrm{mg}$ daily. Although the 
median time to blood culture negativity was shorter at 2 days in the micafungin $100 \mathrm{mg}$ daily and caspofungin $50 \mathrm{mg}$ daily arms versus 3 days in the micafungin $150 \mathrm{mg}$ daily arm, the difference was not statistically significant. This study established the $100 \mathrm{mg}$ daily dose of micafungin as the standard dose for the treatment of serious candidal infections and allows for a cost savings over the $150 \mathrm{mg}$ dose.

Data from the two non-inferiority Phase III trials were pooled for post hoc analyses of various subgroups. ${ }^{42-44}$ Success rates did not differ significantly between micafungin and LAmB/caspofungin in patients with C. glabrata and C. krusei. ${ }^{43}$ Overall treatment success in patients with serious candidal infections, with or without underlying malignancies, was comparable for micafungin and the comparator agents. ${ }^{44}$

A substudy of pediatric patients, including neonates, with candidemia or other types of invasive candidiasis, demonstrated the efficacy of intravenous micafungin in this patient population. ${ }^{45}$ The study consisted of 98 patients in the mITT population. Over $90 \%$ of the patients had candidemia; non-albicans species infected about $60 \%$ of the patients. Micafungin was dosed at $2 \mathrm{mg} / \mathrm{kg}$ daily for those weighing $\leq 40 \mathrm{~kg}$, or $100 \mathrm{mg}$ daily for those weighing $>40 \mathrm{~kg}$. LAmB was dosed at $3 \mathrm{mg} / \mathrm{kg}$ daily. Dosage adjustments were allowed based on efficacy and tolerability. The primary efficacy endpoint was overall success (both clinical and mycological) at the EOT in the mITT population. Overall success occurred in $73 \%(35 / 48)$ of the patients in the micafungin arm and $76 \%$ $(38 / 50)$ in the LAmB arm. Comparable success rates were seen between groups irrespective of patient age, underlying diagnosis, neutropenic status, and presence of a catheter at baseline. Persistence at the EOT was seen in $11 \%(2 / 18)$ of those treated with micafungin and none $(0 / 13)$ of those treated with LAmB for patients infected with C. albicans. For those with non-albicans infections, persistence was seen in $18 \%$ $(5 / 28)$ and $22 \%(7 / 32)$ in the micafungin and LAmB arms, respectively. During the 12-week follow-up period, three patients in the micafungin arm compared with none in the LAmB arm experienced recurrence of infection.

In an open-label, prospective, non-comparative study of micafungin for the treatment of new and refractory candidemia in adults and children, the overall success was $83.3 \%{ }^{46}$ For adults, the success rate was $84.9 \%$, and for pediatric patients it was $75 \%$. Neutropenic patients had a somewhat lower response of $74.2 \%$ overall. For primary therapy, the response rate was $87.5 \%$, and for refractory disease, it was $76 \%$. Combination therapy was allowed for patients with refractory disease. In those patients who received micafungin plus other licensed antifungal therapy, the response rate was
$79.3 \%$. In this study, the dose was $50 \mathrm{mg}$ intravenously daily for C. albicans and $100 \mathrm{mg}$ intravenously daily for nonalbicans species. Pediatric dosing was $1-2 \mathrm{mg} / \mathrm{kg}$ daily. Dose escalation was allowed. Per pathogen success was highest for C. glabrata at $93.8 \%$ and lowest for C. krusei at $63.6 \%$. The response rate by dose range was also examined in this study. A complete or partial response of over $90 \%$ was seen with doses between 75-150 mg/day. For doses between 150 and $200 \mathrm{mg}$ per day, the response rate was only $56 \%$, and for doses over $200 \mathrm{mg}$ per day, the response rate was $67 \%$, although the number of patients who received higher doses was small. The question of a paradoxical effect has been raised for the echinocandins, although the more likely explanation is that sicker patients or patients who were not responding well to therapy were given higher doses.

In summary, micafungin is non-inferior to intravenous caspofungin or LAmB for the treatment of adult and pediatric patients with serious candidal infections. Its efficacy has been high and consistent with the other echinocandins.

\section{Safety and tolerability}

Micafungin was generally well-tolerated by adult and pediatric patients in various clinical trials. Of over 3,000 patients who received micafungin in trials for candidemia and other forms of invasive candidiasis, esophageal candidiasis, invasive aspergillosis, or as prophylaxis after HSCT, approximately one-third experienced a treatment-related adverse effect. ${ }^{47}$ The most frequently reported treatment-related adverse events were nausea $(2.8 \%$ of subjects), elevated alkaline phosphatase $(2.7 \%)$, phlebitis $(2.5 \%)$, vomiting (2.5\%), elevated aspartate aminotransferase levels $(2.3 \%)$, hypokalemia (2.1\%), fever (2.1), and elevated alanine aminotransferase levels (2\%). ${ }^{47}$ Micafungin was well-tolerated by pediatric patients. ${ }^{48}$ The incidence of treatment-related adverse events was lower in pediatric patients. ${ }^{47}$ Although over $90 \%$ of pediatric patients experienced at least one adverse event during treatment, only $2.4 \%$ discontinued treatment because of an adverse event. Twenty-six percent of adverse events that occurred during treatment were considered to be possibly related to the study drug. Micafungin was as well-tolerated as caspofungin and fluconazole, and better tolerated than LAmB and itraconazole,,$^{24,32,33,39,41}$ although pediatric patients, especially those less than 1 year of age, appear more likely than adults to develop abnormalities in liver function tests. ${ }^{47,48}$

A special warning and precaution for use (black box warning) has been issued for micafungin, based on the development of foci of altered hepatocytes and hepatocellular tumors 
after a treatment period $\geq 3$ months that were observed in rats. The assumed threshold for tumor development in rats is in the range of clinical exposure; however, the relevance of this finding in humans is unknown. It is advised that liver function be monitored carefully during treatment and if significant and persistent elevation of aspartate aminotransferase/alanine aminotransferase occurs, it is recommended that therapy be discontinued. For patients with severe liver dysfunction or chronic liver disease, including conditions which predispose to malignancy, micafungin therapy could be based on a careful risk-benefit analysis., ${ }^{7,37}$

\section{Pharmacoeconomic considerations of using micafungin}

Cost considerations in the use of antifungal therapy, in general, and echinocandins in particular, are important. Published literature supports the concept that echinocandins are cost-effective and may be associated with net savings, despite the higher drug acquisition costs when compared with other antifungal therapies. ${ }^{49}$ Several pharmacoeconomic analyses have been published for micafungin. ${ }^{50-56}$ Most of the analyses are based on pharmacoeconomic modeling of data from major clinical trials. Micafungin has been found to be a cost-effective alternative to LAmB. ${ }^{50}$ In this study, treatment costs were lower and effectiveness was higher for micafungin compared with $\mathrm{LAmB}$, leading to the conclusion that micafungin is more cost-effective. Data from the same study were analyzed from an Australian hospital perspective and found that micafungin had a lower total cost than LAmB and a total net cost savings that was primarily due to the lower cost associated with initial antifungal treatment and shorter length-of-stay for patients treated with micafungin. ${ }^{53}$ The cost-effectiveness of micafungin, compared to caspofungin in the treatment of candidemia and other forms of invasive candidiasis, was evaluated using decision analysis with data derived from the Phase III comparative trial. ${ }^{54}$ Total treatment costs were similar for the two agents; however, more patients in the micafungin arm were successfully treated and alive, compared to the caspofungin arm. It was concluded that micafungin is cost-effective compared to caspofungin, although the difference was not significant.

The cost-effectiveness of micafungin as prophylaxis for patients undergoing chemotherapy or HSCT has been studied. ${ }^{51,52,54,55}$ The cost of different antifungal agents for prophylaxis of invasive fungal infections in neutropenic patients in Japan was assessed with a cost simulation model. ${ }^{51}$ Probabilities of prophylaxis failure, possible cases for empiric therapy, probable portions of infections caused by fungal species among prophylaxis failure patients, and incidence of adverse events were based on an analysis of previously reported clinical trials. The antifungal agents studied included oral fluconazole, oral itraconazole, micafungin, and LAmB. In this model, oral itraconazole was the most cost-effective prophylactic antifungal agent in patients who could be given oral therapy. This study had several limitations, including the study design, which limited the range of costs, the possibility of selection bias in the analyzed trials, and the lack of validation of the decision model used with actual clinical practice.

Two studies examined the cost-effectiveness of micafungin versus fluconazole as antifungal prophylaxis for patients undergoing HSCT. ${ }^{54,55}$ Data for these studies was derived from the Phase III randomized, double-blind, comparative trial involving 882 adult and pediatric patients. ${ }^{24}$ Both studies concluded that micafungin was a cost-effective prophylactic antifungal strategy by providing lower medical costs compared with fluconazole prophylaxis.

The use of micafungin for suspected ICU-acquired candidemia among patients with sepsis has been studied in a hypothetical cohort of 1,000 patients. The strategies were either empirical therapy with micafungin, empirical therapy with fluconazole, or no treatment. In this model, treatment with micafungin would result in four fewer deaths and an incremental cost-effectiveness over fluconazole of approximately $\$ 35,000$ per quality-adjusted life-year. The authors concluded that empirical treatment of ICU-acquired candidemia with $100 \mathrm{mg}$ of micafungin daily is a cost-effective alternative to fluconazole, given the increasing likelihood of azole resistance among candidal isolates. ${ }^{56}$

\section{Conclusion}

Micafungin has proven to be a valuable addition to the antifungal armamentarium. Efficacy has been proven in both adult and pediatric patients, including neonates. Its efficacy for prophylaxis in neutropenic patients and the treatment of candidemia and other forms of invasive candidiasis is supported by evidence generated from comparative, randomized clinical trials, and it has been included in multiple clinical practice guidelines. Its role in the treatment of Candida esophagitis remains as salvage therapy in those who are refractory to or intolerant of oral therapy. It has shown efficacy and safety as primary or salvage therapy in high-risk patients with invasive aspergillosis, although the number of patients receiving micafungin monotherapy was small. Although studies supporting a role for micafungin in empirical therapy of febrile neutropenic patients are open- 
label and small numbers, data for caspofungin provide additional support. The safety profile of micafungin is very good and comparable to fluconazole. Pharmacoeconomic studies, based on modeling of data, mainly from large efficacy trials, support the cost-effectiveness of micafungin, mainly by reducing hospital length-of-stay.

\section{Disclosure}

The authors report no conflicts of interest in this work.

\section{References}

1. McNeil MM, Nash SL, Hajjeh RA, et al. Trends in mortality due to invasive mycotic diseases in the United States, 1980-1997. Clin Infect Dis. 2001;33(5):641-647.

2. Wisplinghoff H, Bischoff T, Tallent SM, Seifert H, Wenzel RP, Edmond MB. Nosocomial bloodstream infections in US hospitals: analysis of 24,179 cases from a prospective nationwide surveillance study. Clin Infect Dis. 2004;39(3):309-317.

3. Morrell M, Fraser VJ, Kollef MH. Delaying the empiric treatment of Candida bloodstream infection until positive blood culture results are obtained: a potential risk factor for hospital mortality. Antimicrob Agents Chemother. 2005;49(9):3640-3645.

4. Parkins MD, Sabuda DM, Elsayed S, Laupland KB. Adequacy of empirical antifungal therapy and effect of outcome among patients with invasive Candida species infections. J Antimicrob Chemother. 2007;60(3):613-618.

5. Pfaller MA, Diekema DJ. Epidemiology of invasive candidiasis: a persistent public health problem. Clin Microbiol Rev. 2007;20(1): 133-163.

6. Pappas PG, Kauffman CA, Andes D, et al; Infectious Diseases Society of America. Clinical practice guidelines for the management of candidiasis: 2009 update by the Infectious Diseases Society of America. Clin Infect Dis. 2009;48(5):503-535.

7. Mycamine ${ }^{\circledR}$ (micafungin) [prescribing information]. Northbrook, IL: Astellas Pharma US, Inc; 2012.

8. Denning DW. Echinocandin antifungal drugs. Lancet. 2003;362(9390): 1142-1151.

9. Eschenauer G, DePestel DD, Carver PL. Comparison of echinocandin antifungals. Ther Clin Risk Manag. 2007;3(1):71-97.

10. Baddley JW, Moser SA. Emerging fungal resistance. Clin Lab Med. 2004;24(3):721-735, vii.

11. Park S, Kelly J, Kahn JN, et al. Specific substitutions in the echinocandin target Fksp1 account for reduced susceptibility of rare laboratory and clinical Candida species isolates. Antimicrob Agents Chemother. 2005;49(8):3264-3273.

12. Pfaller MA, Diekema DJ. Progress in antifungal susceptibility testing of Candida spp. by use of Clinical and Laboratory Standards Institute broth microdilution methods, 2010 to 2012. J Clin Microbiol. 2012;50(9): 2846-2856.

13. Hebert MF, Smith HE, Marbury TC, et al. Pharmacokinetics of micafungin in health volunteers, volunteers with moderate liver disease, and volunteers with renal dysfunction. J Clin Pharmacol. 2005;45(10): $1145-1152$.

14. Tabata K, Katashima M, Kawamura A, Kaibara A, Tanigawara Y. Population pharmacokinetic analysis of micafungin in Japanese patients with fungal infections. Drug Metab Pharmacokinet. 2006;21(4): 324-331.

15. Seibel NL, Schwartz C, Arrieta A, et al. Safety, tolerability, and pharmacokinetics of micafungin (FK463) in febrile neutropenic pediatric patients. Antimicrob Agents and Chemother. 2005;49(8): 3317-3324.

16. Hall RG, Swancutt MA, Gumbo T. Fractal geometry and the pharmacometrics of micafungin in overweight, obese, and extremely obese people. Antimicrob Agents Chemother. 2011;55(11):5107-5112.
17. Walsh TJ, Goutelle S, Jelliffe RW, et al. Intrapulmonary pharmacokinetics and pharmacodynamics of micafungin in adult lung transplant recipients. Antimicrob Agents Chemother. 2010;54(8): 3451-3459.

18. Cateau E, Rodier MH, Imbert C. In vitro efficacies of caspofungin or micafungin catheter lock solutions on Candida albicans biofilm growth. J Antimicrob Chemother. 2008;62(1):153-155.

19. Jacobson MJ, Steckelberg KE, Piper KE, Steckelberg JM, Patel R. In vitro activity of micafungin against planktonic and sessile Candida albicans isolates. Antimicrob Agents Chemother. 2009;53(6):2638-2639.

20. Simitsopoulou M, Peshkova P, Tasin E, et al. Species-specific and drug-specific differneces in susceptibility of Candida biofilms to echinocandins : characterization of less common bloodstream isolates. Antimicrob Agents Chemother. 2013;57(6):2562-2570.

21. Copelan EA. Hematopoietic stem-cell transplantation. N Engl J Med. 2006;354(17):1813-1826.

22. Goodman JL, Winston DJ, Greenfield, RA, et al. A controlled trial of fluconazole to prevent fungal infections in patients undergoing bone marrow transplantation. $N$ Engl J Med. 1992;326(13):845-851.

23. Slavin MA, Osborne B, Adams R, et al. Efficacy and safety of fluconazole prophylaxis for fungal infections after marrow transplantation - a prospective, randomized, double-blind study. $J$ Infect Dis. 1995;171(6):1545-1552.

24. van Burik JA, Ratanatharathorn V, Stepan, DE, et al; National Institute of Allergy and Infectious Diseases Mycoses Study Group. Micafungin versus fluconazole for prophylaxis against invasive fungal infections during neutropenia in patients undergoing hematopoietic stem cell transplantation. Clin Infect Dis. 2004;39(10):1407-1416.

25. Freifeld AG, Bow EJ, Sepkowitz KA, et al. Clinical practice guidelines for the use of antimicrobial agents in neutropenic patients with cancer: 2010 update by the Infectious Diseases Society of America. Clin Infect Dis. 2011;52(4):e56-e93.

26. Prentice HG, Kibbler CC, Prentice AG. Towards a targeted, riskbased, antifungal strategy in neutropenic patients. Br J Haematol. 2000;110(2):273-284.

27. Toubai T, Tanaka J, Ota S, et al. Efficacy and safety of micafungin in febrile neutropenia patients treated for hematological malignancies. Intern Med. 2007;46(1):3-9.

28. Goto N, Hara T, Tsurumi H, et al. Efficacy and safety of micafungin for treating febrile neutropenia in hematological malignancies. $\mathrm{Am} \mathrm{J}$ Hematol. 2010;85(4):872-876.

29. Barbaro G, Barbarini G, Di Lorenzo G. Fluconazole compared with itraconazole in the treatment of esophageal candidiasis in AIDS patients: a double-blind, randomized, controlled clinical study. Scand J Infect Dis. 1995;27(6):613-617.

30. Pettengell K, Mynhardt J, Kluyts T, Lau W, Facklam D, Buell D; FK463 South African Study Group. Successful treatment of oesophageal candidiasis by micafungin: a novel systemic antifungal agent. Aliment Pharmacol Ther. 2004;20(4):475-481.

31. Fichtenbaum CJ, Koletar S, Yiannoutsos C, et al. Refractory mucosal candidiasis in advanced human immunodeficiency virus infection. Clin Infect Dis. 2000;30(5):749-756.

32. de Wet N, Llanos-Cuentas A, Suleiman J, et al. A randomized, doubleblind, parallel-group, dose-response study of micafungin compared with fluconazole for the treatment of esophageal candidiasis in HIV-positive patients. Clin Infect Dis. 2004;39(6):842-849.

33. de Wet NTE, Bester AJ, Viljoen JJ, et al. A randomized, double blind, comparative trail of micafungin (FK463) vs fluconazole for the treatment of oesophageal candidiasis. Aliment Pharmacol Ther. 2005;21(7):899-907.

34. Walsh TJ, Anaissie EJ, Denning DW, et al; Infectious Diseases Society of America. Treatment of aspergillosis: Clinical practice guidelines of the Infectious Diseases Society of America. Clin Infect Dis. 2008;46(3): 327-360.

35. Denning DW, Marr KA, Lau WM, et al. Micafungin (FK463), alone or in combination with other systemic antifungal agents, for the treatment of acute invasive aspergillosis. J Infect. 2006;53(5):337-349. 
36. Kontoyiannis DP, Ratanatharathorn V, Young JA, et al. Micafungin alone or in combination with other antifungal therapies in hematopoietic stem cell transplant recipients with invasive aspergillosis. Transpl Infect Dis. 2008;11(1):89-93.

37. Mycamine ${ }^{\circledR}$ (micafungin) [European Medicine Agency prescribing information]. London: European Medicines Agency; 2013. Available from: www.ema.europa.eu/docs/en. Accessed November 13, 2013.

38. Andes DR, Safdar N, Baddley JW; Mycoses Study Group. Impact of treatment strategy on outcomes in patients with candidemia and other forms of invasive candidiasis: a patient-level quantitative review of randomized trials. Clin Infect Dis. 2012;54(8):1110-1122.

39. Kuse, ER, Chetchotisakd P, da Cunha C, et al; Micafungin Invasive Candidiasis Working Group. Micafungin versus liposomal amphotericin B for candidaemia and invasive candidosis: a phase III randomized double-blind trial. Lancet 2007;369(9572):1519-1527.

40. Dupont BF, Lortholary O, Ostrosky-Zeichner, Stucker F, Yeldandi V. Treatment of candidemia and invasive candidiasis in the intensive care unit: post hoc analysis of a randomized, controlled trial comparing micafungin and liposomal amphotericin B. Crit Care. 2009;13(5):R159.

41. Pappas PG, Rotstein F, Betts RF, et al. Micafungin versus caspofungin for treatment of candidemia and other forms of invasive candidiasis. Clin Infect Dis. 2007;45(7):883-893.

42. Nucci M, Anaissie E, Betts RF, et al. Early removal of central venous catheter in patients with candidaemia does not improve outcome: analysis of 482 patients from 2 randomized clinical trials. Clin Infect Dis. 2010;51(3):295-303.

43. Shorr AF, Wu C, Kothari S. Outcomes with micafungin in patients with candidaemia or invasive candidiasis due to Candida glabrata and Candida krusei. J Antimicrob Chemother. 2011;(66):375-380.

44. Cornely OA, Marty FM, Stucker F, Pappas PG, Ullmann AJ. Efficacy and safety of micafungin for treatment of serious Candida infections in patients with or without malignant disease. Mycoses. 2011;54(6):e838-e847.

45. Queiroz-Telles F, Berezin E, Leverger G, et al; Micafungin Invasive Candidiasis Study Group. Micafungin versus liposomal amphotericin B for pediatric patients with invasive candidiasis: substudy of a randomized double-blind trial. Ped Infect Dis. 2008;27(9):820-826.

46. Ostrosky-Zeichner L, Kontoyiannis D, Raffalli J, et al. International, open-label, noncomparative, clinical trial of micafungin alone and in combination for treatment of newly diagnosed and refractory candidemia. Eur J Clin Microbiol Infect Dis. 2005;24(10):654-661.
47. Cornely OA, Pappas PG, Young JA, Maddison P, Ullmann AJ. Accumulated safety data of micafungin in therapy and prophylaxis in fungal diseases. Expert Opin Drug Saf. 2011;10(2):171-183.

48. Arrieta AC, Madison P, Gross AH. Safety of micafungin in pediatric clinical trials. Pediatr Infect Dis J. 2011;30(6):e97-e102.

49. Wilke M. Treatment and prophylaxis of invasive candidiasis with anidulafungin, caspofungin and micafungin and its impact on use and costs: review of the literature. Eur J Med Res. 2011;16(4):180-186.

50. Cornely OA, Sidhu M, Odeyemi I, van Engen AK, van der Wall JM, Schoeman O. Economic analysis of micafungin versus liposomal amphotericin B for treatment of candidaemia and invasive candidiasis in Germany. Curr Med Res Opin. 2008;24(6):1743-1753.

51. Imataki O, Kubota Y, Ohnishi H, Kitanaka A, Ishida T, Tanaka T. Medical cost analysis for antifungal prophylaxis in neutropenic patients with hematological malignancies: a systematic simulation analysis. Support Care Cancer. 2011;19(10):1657-1665.

52. Neoh CF, Liew D, Slavin M, et al. Economic evaluation of micafungin vs Liposomal Amphotericin B (LAmB) for the treatment of candidaemia and Invasive Candidiasis (IC). Mycosis. 2013;56(5):532-542.

53. Sidhu MK, van Engen, AK, Kleintjens J, Schoeman O, Palazzo M. Cost-effectiveness analysis of micafungin versus caspofungin for treatment of systemic Candida infections in the UK. Cur Med Res Opin. 2009;25(8):2049-2059.

54. Schonfeld W, Wang Cheng J, Tong KB, Seifeldin R. Cost-effectiveness analysis of antifungal prophylaxis in patients undergoing hematopoietic stem cell transplantation. Clin Ther. 2008;30(5):964-973.

55. Sohn HS, Lee TJ, Kim J, Kim D. Cost-effectiveness analysis of micafungin versus fluconazole for prophylaxis of invasive fungal infections in patients undergoing hematopoietic stem cell transplantation in Korea. Clin Ther. 2009;31(5):1105-1115; discussion 1066-1068.

56. Zilberberg MD, Kothari S, Shorr AF. Cost-effectiveness of micafungin as an alternative to fluconazole empiric treatment of suspected ICUacquired candidemia among patients with sepsis: a model simulation Crit Care. 2009;13(3):R94.
Core Evidence

\section{Publish your work in this journal}

Core Evidence is an international, peer-reviewed open-access journal evaluating the evidence underlying the potential place in therapy of drugs throughout their development lifecycle from preclinical to postlaunch. The focus of each review is to evaluate the case for a new drug or class in outcome terms in specific indications and patient groups.

\section{Dovepress}

The manuscript management system is completely online and includes a very quick and fair peer-review system, which is all easy to use. Visit http://www.dovepress.com/testimonials.php to read real quotes from published authors. 